\title{
Eye Movements during Visuomotor Adaptation Represent Only Part of the Explicit Learning
}

\author{
Zohar Bromberg, ${ }^{1,2}$ (D) Opher Donchin, ${ }^{1,2}$ and ${ }^{-S h l o m i ~ H a a r ~}{ }^{3}$
}

https://doi.org/10.1523/ENEURO.0308-19.2019

${ }^{1}$ Department of Biomedical Engineering, Ben-Gurion University of the Negev, Beer-Sheva, 8410501 Israel, ${ }^{2}$ Zlotowski Center for Neuroscience, Ben-Gurion University of the Negev, Beer-Sheva, 8410501 Israel, and ${ }^{3}$ Department of Bioengineering, Imperial College London, London SW7 2AZ, United Kingdom

\begin{abstract}
Visuomotor rotations are learned through a combination of explicit strategy and implicit recalibration. However, measuring the relative contribution of each remains a challenge and the possibility of multiple explicit and implicit components complicates the issue. Recent interest has focused on the possibility that eye movements reflects explicit strategy. Here we compared eye movements during adaptation to two accepted measures of explicit learning: verbal report and the exclusion test. We found that while reporting, all subjects showed a match among all three measures. However, when subjects did not report their intention, the eye movements of some subjects suggested less explicit adaptation than what was measured in an exclusion test. Interestingly, subjects whose eye movements did match their exclusion could be clustered into the following two subgroups: fully implicit learners showing no evidence of explicit adaptation and explicit learners with little implicit adaptation. Subjects showing a mix of both explicit and implicit adaptation were also those where eye movements showed less explicit adaptation than did exclusion. Thus, our results support the idea of multiple components of explicit learning as only part of the explicit learning is reflected in the eye movements. Individual subjects may use explicit components that are reflected in the eyes or those that are not or some mixture of the two. Analysis of reaction times suggests that the explicit components reflected in the eye movements involve longer reaction times. This component, according to recent literature, may be related to mental rotation.
\end{abstract}

Key words: visuomotor rotation; explicit learning; eye movement; motor control; motor learning; motor adaptation

\section{Significance Statement}

Visuomotor adaptation involves both explicit and implicit components: aware reaiming and unaware error correction. Recent studies suggest that eye movements could be used to capture the explicit component, a method that would have significant advantages over other approaches. We show that eye movements capture only one component of explicit adaptation. This component scales with reaction time while the component unrelated to eye movements does not. Our finding has obvious practical implications for the use of eye movements as a proxy for explicit learning. However, our results also corroborate recent findings suggesting the existence of multiple explicit components, and, specifically, their decomposition into components correlated with reaction time and components that are not.

\section{Introduction}

Visuomotor adaptation is commonly used to study human motor learning in health (Krakauer et al., 1999;

Received August 3, 2019; accepted November 9, 2019; First published November 27, 2019.

The authors declare no competing financial interests.
Ghilardi et al., 2000; Taylor and Ivry, 2013; Galea et al., 2015; Haar et al., 2015b) and disease (Rabe et al., 2009; Wong et al., 2019). In a visuomotor rotation task, the 
visual representation of hand position is manipulated such that subjects must learn a new mapping of motor commands to apparent outcomes. Recent studies dissociated explicit and implicit processes in the visuomotor adaptation (Mazzoni and Krakauer, 2006; Hegele and Heuer, 2010; Taylor and Ivry, 2011; Taylor et al., 2014; Werner et al., 2015), where the sum of the two gives the total adaptation.

One measure of implicit learning is to ask subjects to reach straight for the target without perturbation (or without any visual feedback) and to measure the difference between the direction of reach and the target. We call this exclusion because the subject is being asked to "exclude" their explicit knowledge from their behavior. When measured after adaptation, this is called aftereffect. During adaptation, it is sometimes called a "catch trial" (Werner et al., 2015). Exclusion cannot be measured every trial since it presumes surrounding adaptation trials. To assess implicit and explicit learning throughout the adaptation process, Taylor et al. (2014) suggested simply asking subjects to report aiming direction before each movement by reporting which of the numbers displayed in a circle on the screen was in the direction the subject intended to move. Reporting has been a very productive experimental approach. However, the protocol has known limitations [e.g., reporting increases the length and variability of reaction time (RT) since subjects can start moving only after reporting].

One alternative is to measure explicit learning using eye movements: perhaps eye movements can provide an objective measure of subjects' intentions without needing special trials or direct questioning. During unperturbed reaching movements, the eyes were found to provide an unbiased estimate of hand position (Ariff et al., 2002). During visuomotor rotation, there is an increase in correlation between gaze and hand directions in early practice, which gradually decreased thereafter (Rand and Rentsch, 2016). Indeed, a recent study found that gaze patterns during visuomotor adaptation were linked to explicit learning (de Brouwer et al., 2018). Interestingly, de Brouwer et al. (2018) noticed subjects whose eye movements did not reflect adaptation while their aftereffects did indicate some explicit learning. This raises the possibility that some forms of explicit adaptation are captured by the eye movements, while others are not.

This possibility is in line with recent suggestions of multiple explicit strategies in human motor learning, even in a redundant visuomotor rotation task (McDougle and Taylor, 2019). McDougle and Taylor (2019) showed that

This work was partially supported by Deutsche Forschungsgemeinschaft Grant TI-239/16-1 (O.D.). S.H. is supported by the Royal Society-Kohn International Fellowship (Grant NF170650).

Acknowledgments: We thank Ilan Dinstein and Ayelet Arazi for help with the eye tracking.

Correspondence should be addressed to Shlomi Haar at s.haar@imperial.ac.uk.

https://doi.org/10.1523/ENEURO.0308-19.2019

Copyright (C) 2019 Bromberg et al.

This is an open-access article distributed under the terms of the Creative Commons Attribution 4.0 International license, which permits unrestricted use, distribution and reproduction in any medium provided that the original work is properly attributed. subjects in different conditions may use either discrete response caching or parametric mental rotation as two different explicit strategies. Their results further suggest that RT can be used to dissociate these explicit strategies: mental rotation is a time-consuming computation, and caching is a fast automatic process that does not require a long RT (Haith and Krakauer, 2018). Here, we explore the explicit components captured by eye movements and their link to the explicit strategies captured by RT.

In the first experiment of the current study, we measured subjects' eye movements during visuomotor rotation with verbal reporting and without. As in de Brouwer et al. (2018), our results demonstrate that, in verbal reporting, eye fixations before movement onset accurately predict the reported aiming direction. Without reporting, eye fixation before movement onset correlates well with explicit learning measured by aftereffect. However, it does not account for the full explicit knowledge revealed by exclusion. This suggests that only a component of explicit learning is being captured by eye movements when there is no verbal report.

In a second experiment, we explored the time course of the discrepancy between eye movements and exclusion by introducing exclusion (catch) trials, during adaptation, in addition to testing for an aftereffect at the end of adaptation. For some subjects, measures of explicit learning from eye movements matched those from exclusion. For other subjects, exclusion revealed more explicit knowledge than that found in the eye movements. The first group was divided into the following two subgroups: those using primarily an explicit strategy and those with hardly any contribution from an explicit strategy. The second group, where exclusion showed more explicit knowledge than did the eye movements, showed subjects with the full range of combinations of explicit and implicit learning. Further analysis of RT seems to indicate that the explicit knowledge reflected in the eye movements may be the same mental rotation component identified by McDougle and Taylor (2019).

\section{Materials and Methods}

\section{Participants}

One hundred fourteen right-handed subjects with normal or corrected-to-normal vision participated in the study: 44 subjects ( 31 females; age, 18-29 years) in the first experiment and 70 subjects ( 46 females; age, $18-31$ years) in the second experiment. Of these, five subjects were excluded due to sparse eye movement data (four subjects from the first experiment and one subject from the second), and three subjects were excluded from the second experiment because they misunderstood the exclusion instructions (details below). All subjects signed an informed consent form, which also asked for basic information about their relevant medical status. None of the subjects reported neurologic or motor impairments. The experimental protocol was approved by the Human Subject Research Committee of Ben-Gurion University of the Negev. 

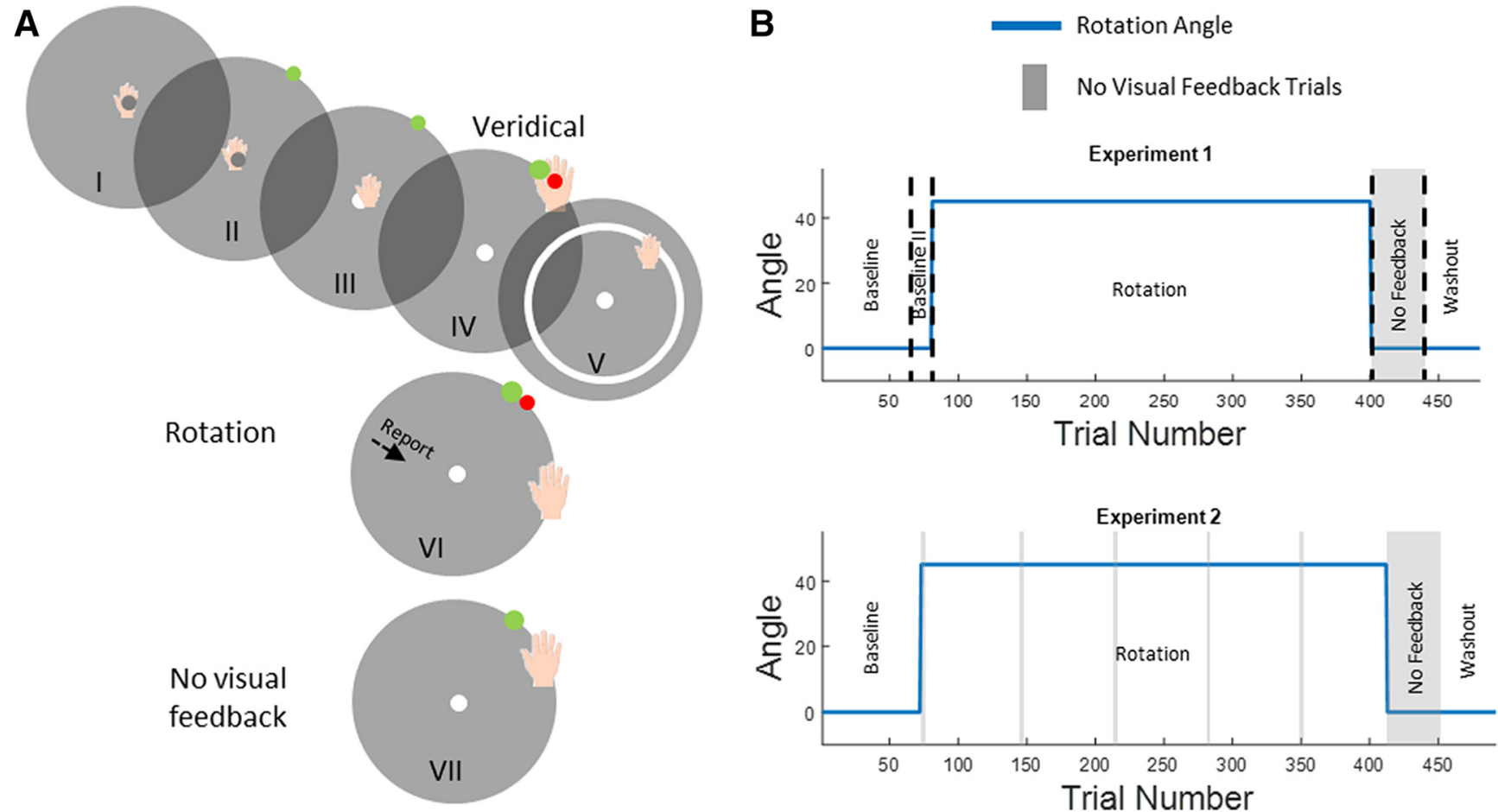

Figure 1. Trial and experiment design. A, Basic trial structure. Subjects performed reaching movements from the origin (I). After $1 \mathrm{~s}$, a target appeared (II), and the subject started reaching toward the target (III). On veridical trials, when reaching to target, a red circle appeared indicating the hand position (IV). After the feedback disappeared, a white ring appeared, which directed the hand back to the origin $(\mathrm{V})$. In rotation trials, the red circle appeared rotated $45^{\circ}$ counterclockwise $(\mathrm{VI})$. In the report condition, subjects were asked to report before initiating their hand movement. In no visual feedback trials, subjects received no feedback at the end of the movement (VII). B, Experimental design. Top, First experiment. In the first and second baseline block, feedback was veridical. In the rotation block, the cursor was rotated by $45^{\circ}$. In the no visual feedback block, the landmarks and cursor feedback were removed, and participants were instructed to aim directly at the target. In the washout block, conditions were similar to the first baseline block. In the second baseline block and in the rotation block, participants in the R condition reported their aiming direction. Bottom, Second experiment. Similar to the first experiment, however, without the second baseline block and without report sessions. In addition, five mini exclusion blocks were spread throughout the rotation block.

\section{Apparatus}

Participants were comfortably seated on a heightadjustable chair at a table with a digitizing Wacom Intuos Pro tablet with an active area of $311 \times 216 \mathrm{~mm}$ and a polling rate of $2154 \mathrm{~Hz}$. A box was used to hide a subject's hand and the tablet as they faced a 19 inch vertical computer screen. Their eye movements were recorded with an EyeLink 1000 (SR Research) eyetracking system, with sampling rate of $500 \mathrm{~Hz}$ and the accuracy of $\sim 0.5^{\circ}$. The participants rested their head on a stabilizing support which included braces for the chin and forehead. Calibration of the eye-tracking system was performed for each subject before the experiment. During the experiment, subjects made centerout, horizontal reaching movements on the surface of the tablet using a digital stylus. On the monitor, they saw a cursor and targets to move to (detailed in the next section). The movements of the cursor matched the movements of the tablet pen (except as detailed in the next section). The EyeLink system provides the location of the pupil, and the timing and location of events including blinks, fixations, and saccades. In the reporting group (first experiment), the number that subjects reported as their intended aiming direction was recorded and typed into an Excel spreadsheet (details below).

\section{Trial structure}

We used three different trial types in our experiments. The general structure of these trial types is shown in Figure $1 A$. At the beginning of every trial, subjects moved a gray cursor to a white origin. The origin appeared in the center of the screen and corresponded to a hand position at the center of the tablet. After maintaining this position for $1000 \mathrm{~ms}$, a green target circle appeared at a distance of $8 \mathrm{~cm}$ on the screen, corresponding to a movement of $5.5 \mathrm{~cm}$ on the tablet (generally in the text, we will report distances on the screen and equivalent distances on the tablet will always be 0.6875 of screen distances). Visual landmarks also appeared surrounding the target. Targets could be in one of the eight cardinal and intercardinal directions relative to the origin. The origin and the target sizes on the screen were 1 and $0.5 \mathrm{~cm}^{2}$, respectively. The order of targets was pseudorandom for each subject and between subjects, such that a complete circuit of the targets was completed every eight movements. The cursor disappeared when the participant's hand was further than $0.56 \mathrm{~cm}$ from the center of the origin. When the hand 
crossed the distance of $7.6 \mathrm{~cm}(95 \%$ of the target distance) from the origin, a red circle the same size as the original cursor appeared on the screen at the same distance as the target. This circle was presented to give subjects visual feedback about the cursor position at the end of the trial. The red circle that indicated the cursor location remained visible for 350,700 , or 1000 ms. Different presentation times were used in different groups and in different experiments (detailed below). After the red circle disappeared, a white ring appeared centered at the origin with radius equal to the distance of the hand from the origin. This ring guided the hand back to the origin without providing information about its exact location.

The three different trial types were as follows: veridical feedback trials where the red circle appearing at the end of each trial reflected the true location of the hand; rotation trials where the red circle appeared at a location that was rotated by $45^{\circ}$ counterclockwise relative to the direction of hand movement; and no visual feedback trials where no red circle or any other landmarks appeared at all. In no visual feedback trials, subjects were instructed to aim straight toward the target. In all trials, subjects received auditory feedback to control movement speed: movements that reached a distance of $7.6 \mathrm{~cm}$ from the origin center within 500 ms were rewarded with a pleasant "ding" sound; otherwise, subjects heard an unpleasant "buzz" sound.

\section{Data collection}

The $x$ and $y$ coordinates of the hand trajectories were collected by the tablet and saved from the moment of target appearance until the hand reached a distance of $7.6 \mathrm{~cm}$ from the origin. Eye movements were recorded continuously using the eye tracker. Eye movement data were preprocessed to remove blinks (as recorded by the eye-tracker software). In addition, eye movements were recentered to correct for drift over the experiment by assuming that the eye is fixated on the origin during the $1000 \mathrm{~ms}$ before the hand movement begins. Recentering was accomplished by accumulating eye position in this time window during the current trial, two preceding trials, and two following trials, and taking the median position across the entire $5000 \mathrm{~ms}$ of data.

\section{Experiment 1}

Groups

This experiment had two groups of 22 subjects each: Report and No-Report groups. Each subject was assigned to one of two feedback times: 350 or $700 \mathrm{~ms}$, counterbalanced between the groups. The feedback time showed no effect on task performance, learning, and eye movements in either of the groups, and thus data from both feedback times is combined throughout the article.

\section{Procedure}

Each trial presented one of the eight targets in cardinal and intercardinal directions $\left(0^{\circ}, 45^{\circ}, 90^{\circ}, 135^{\circ}, 180^{\circ}, 225^{\circ}\right.$, $270^{\circ}$, and $315^{\circ}$ ) relative to the origin. For subjects in the Report group, 63 numbered landmarks spaced by $5.625^{\circ}$ appeared on a circle with a radius $8 \mathrm{~cm}$ around the origin (the same distance from the origin as the target). Low numbers were nearer the target. Before each movement, subjects were instructed to say out loud the number toward which they were aiming to get the cursor to the target. For subjects in the No-Report group, hollow circles were presented instead of the numbered landmarks, and they were not asked to report their intended aiming direction. Indeed, the No-Report group was not informed in any way that they might want to aim to a direction different from the target. The experimental sequence was the same for both groups. Each session was divided into five blocks: two baseline blocks (72 and 8 trials) consisting of veridical trials. The first baseline (veridical feedback) block allowed subjects to get familiar with the reaching task, and the second block was intended for subjects in the Report group to practice the report. For subjects in the No-Report group, there was no difference between these two blocks. In the third block, the rotation block (320 rotation trials), the cursor was rotated relative to the origin. Subjects in the Report group were required to report their aiming direction during this block. The fourth block was a nofeedback block (40 trials), which consisted of no visual feedback trials. In the last block, a washout block (40 trials), subjects were presented with veridical feedback trials (Fig. 1B). The percentage of successful trials was displayed at the end of each block. A trial was considered successful if the red circle was within $5^{\circ}$ of the target. Every 40 trials, the experimental program displayed a full-screen text message reminding subjects of the instructions.

\section{Experiment 2 \\ Procedure}

Each trial presented one of the eight targets in secondary intercardinal directions $\left(22.5^{\circ}, 67.5^{\circ}, 112.5^{\circ}, 157.5^{\circ}\right.$, $202.5^{\circ}, 247.5^{\circ}, 292.5^{\circ}$, and $337.5^{\circ}$ ) relative to the origin. In this experiment, trial feedback was visible for $1000 \mathrm{~ms}$. Forty-seven hollow circles spaced $7.5^{\circ}$ apart appeared on the target circle as landmarks surrounding the target before each movement. The experiment was divided into the following four blocks: a baseline block with 72 veridical feedback trials, a rotation block with 320 rotation trials, a no feedback block with 40 no visual feedback trials, and the last block, a washout block with 40 veridical feedback trials. Subjects did 20 additional no visual feedback trials during the rotation block (Fig. 1B). These were evenly spaced during the block in five mini-blocks of four trials each. Subjects were instructed at the beginning of the experiment that their goal is to hit the target with the cursor. All instructions that were given during the experiment were presented on the screen. After the first two trials of the rotation block, a message appeared on the screen asking the subject to pay attention to the error and to hit the target with the cursor. In addition, before and after each mini-block of no visual feedback trials, a message appeared announcing the beginning and end of this block. In the beginning, the message instructed subjects to ignore their strategy and to hit the target. In the end, the message instructed them to go back to using their strategy. 
A
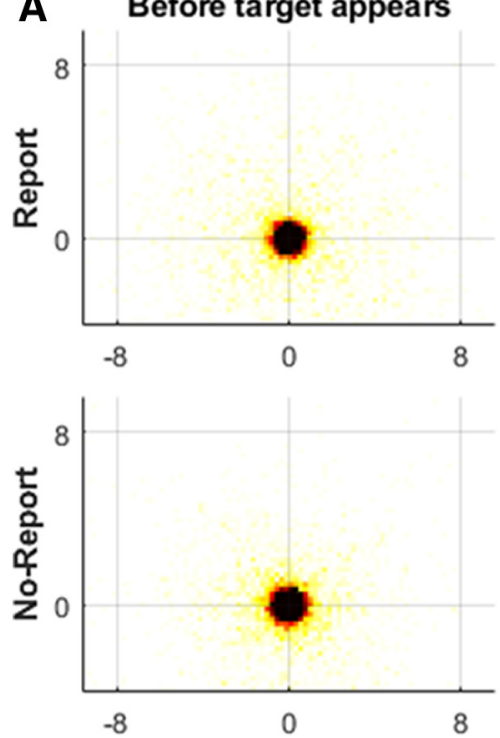

B

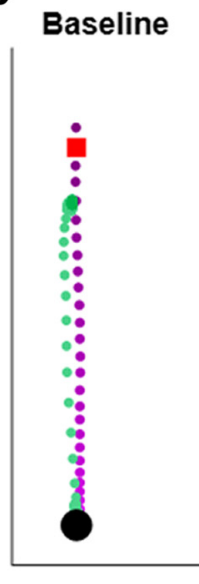

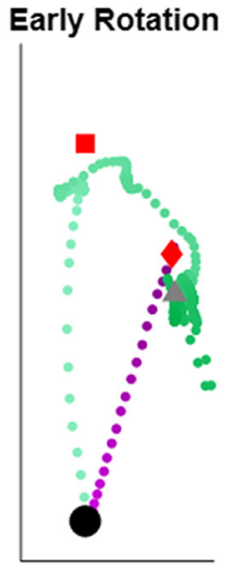

Hand Trajectory
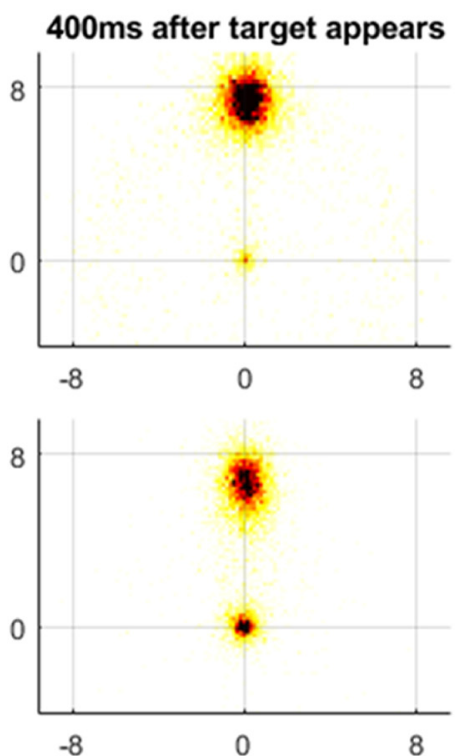

Before hand movement
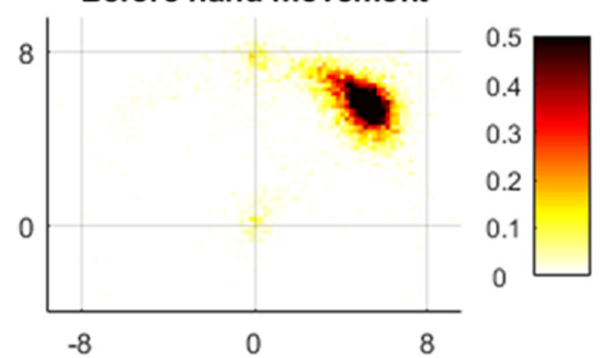

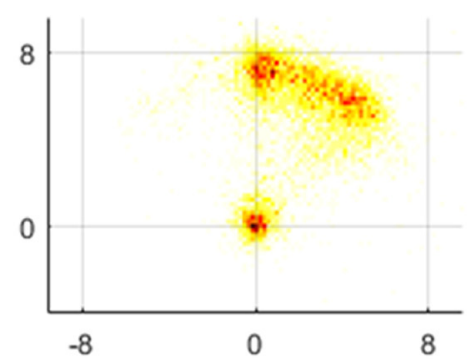

C

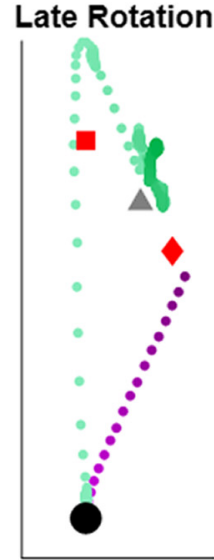

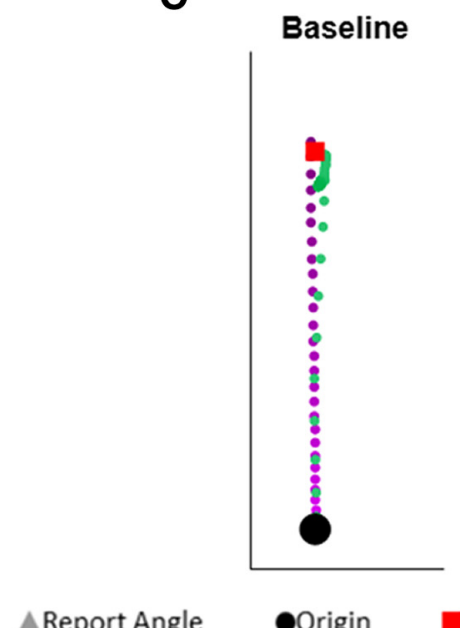

$\triangle$ Report Angle

Eye Trajectory
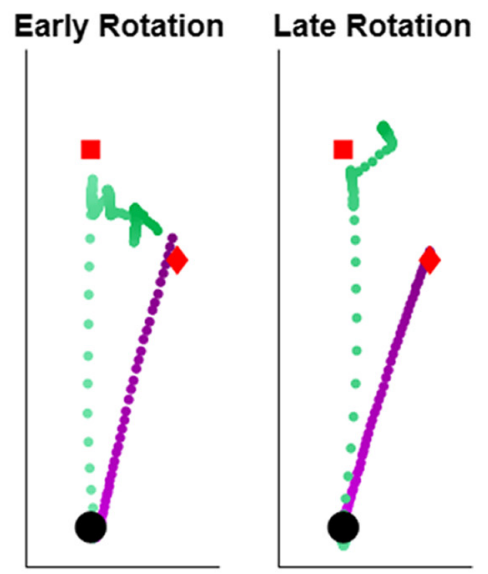

Figure 2. Eye movements pattern. $\boldsymbol{A}$, Fixations distributions during the phases of the trial before movement initiation, in rotation trials: the last fixation before target appears, the point of fixation $400 \mathrm{~ms}$ after, and the last fixation before movement initiation. Report group on top row and no-report on bottom row. Numbers on the axis indicate the distance in centimeters on the screen, $8 \mathrm{~cm}$ from the origin to the targets. $\boldsymbol{B}, \boldsymbol{C}$, Hand and eye trajectories of two individuals from the report $(\boldsymbol{B})$ and the no-report $(\boldsymbol{C})$ groups. In the baseline block, the subjects first gazed toward the target and later made a reaching movement toward it. At the beginning of the rotation block, both subjects shifted their gaze toward the target and later toward the Hand-Target, the reporting subject then reported a direction close to the Hand-Target, and both subjects moved their hand toward the Hand-Target. By the end of the rotation, the subjects also shifted their gaze first to the target, then toward the side opposite of the rotation. However, this secondary shift was smaller than the Hand-Target angle for the reporter and even smaller for the nonreporter. The reporter also reported a smaller angle. Both kept moving their hand to the Hand-Target direction.

\section{General data analysis and statistics Hand movement analysis}

The Hand-Target difference was calculated as the difference between the target location and the hand position when the cursor reached a distance of $7.6 \mathrm{~cm}$ from the origin. Trials in which the movement from the origin toward the target was not strictly increasing after the cursor passed a distance of $0.4 \mathrm{~cm}$ from the origin or trials in which movement was too slow were excluded. In the first experiment, subjects were presented with text reminding them of the instructions every 40 trials. Trials immediately following these reminders were discarded, since subjects often tested the degree of rotation by aiming directly at the target. Each movement RT was defined as the time between target appearance and the cursor reaching $7 \%$ of the target distance.

\section{Eye movement analysis}

The eye movements before movement initiation followed a stereotypical pattern, as follows: during the baseline block, subjects fixated first on the origin and then on the target. During the rotation block, target fixation was often followed by eye movements that carried the gaze in the direction opposite to the rotation (Fig. 2). We tested 
Table 1: Glossary

Hand-Target difference
Explicit report
Implicit report
Explicit eye
Implicit eye
Implicit exclusion
Explicit exclusion

Hand-Target difference

Explicit report

Implicit report

Implicit eye

Explicit exclusion
Angular difference between the target and the hand at the end of the reaching
Reported direction of movement
Hand-Target difference minus explicit report
The angle between the target and the last fixation before movement onset
Hand-Target difference minus explicit eye
The Hand-Target difference during exclusion trials
Hand-Target difference before catch trials minus implicit exclusion several measures of this latter gaze shift to see which best correlated with subjects' reported aiming direction. All methods produced similar results and the choice of measure did not influence our findings. Thus, following previous results showing the eye leading upcoming hand movements with a similar constant lead (Ariff et al., 2002), we choose to use the last fixation before movement onset to characterize subjects' intended aiming direction and called it the "explicit eye." If eye fixation before movement onset was missing (due to a blink) or near the origin (closer than $4 \mathrm{~cm}$ ) or beyond the target area (farther than $12 \mathrm{~cm}$ from the origin), then eye movements for that trial were discarded. Any subject for whom more than half of the rotation trials were discarded was excluded from further analysis (four subjects in the No-Report group in the first experiment and one subject in the second experiment were excluded because too much eye data were discarded). We used the term "implicit eye" for the difference between the explicit eye and the Hand-Target difference; it represents the estimated implicit adaptation derived using the eye movements (Table 1).

\section{Reporting analysis}

Report trials (in the first experiment) were also characterized by the subject's statement of the intended aim direction. The reported number was multiplied by $5.625 \%$ landmark to convert it to an aiming angle. This was called the "explicit report." The "implicit report" was calculated by subtracting the explicit report from the Hand-Target difference. This is the difference between where subjects said they aim and where they moved their hand (Table 1).

\section{No visual feedback trials analysis}

In the no visual feedback trials, subjects were instructed to aim directly toward the target. Thus, the difference between the hand and the target in these trials represents, by definition, residual implicit knowledge of the rotation not under the subject's control. In the second experiment, there were 20 no visual feedback trials in mini-blocks during the rotation block as well as a no visual feedback block after the rotation block. The mini-block trials and the first four trials of the no visual feedback block were named "implicit no visual feedback." We excluded three subjects who later reported they did not understand the instruction during those trials.

\section{Statistics}

Unless otherwise noted, reported values are mean and SEM. A nonparametric bootstrap with 10,000 samples was used to generate the sampling distribution for group means. The 5th and 95th percentiles of the sampling distribution were used to determine the confidence inter- vals reported in the Extended Data. We forego reporting statistical significance as per the recommendations from Amrhein et al. (2019). Calculations of traditional hypothesis tests and effect sizes can also be found in the Extended Data.

The measures defined for each subject and each trial were the difference between the hand and the target (Hand-Target difference), the last fixation before movement onset (explicit eye), and the RT. In the first experiment, the report group also has the reported angle of the aim direction (explicit report). For each measure, we smoothed the results by averaging over the bins of eight sequential trials (one for each target). For each bin, trials that were more than two SDs from the mean of the bin were removed (in the first experiment, $2.0 \%$ of the available Hand-Target difference trials were outliers and $2.3 \%$ from the available explicit eye trials; in the second experiments, $1.9 \%$ and $2.3 \%$ of trials were removed, respectively). We then calculated the implicit measures for each trial by subtracting the corresponding explicit measures (eye and report) from the Hand-Target difference to create the implicit eye and implicit report. In case of a missing value in an explicit measure (due to outliers, slow movements, blinking, missed report, or other reason), no implicit measure was calculated for that measure for that trial. For each measure, we used a parametric bootstrap to find the sampling distribution of the mean for each bin, resampling data from a normal distribution with the outlier-corrected mean and SD determined by the eight points in each bin for each measure (or fewer points where there were missing values).

In the second experiment, we also measured "implicit exclusion," the Hand-Target difference during exclusion trials. We averaged over the four sequential trials of each mini-block to create a binned version of implicit exclusion, and again used a parametric bootstrap to find the sampling distribution of the mean of each bin, resampling data from a normal distribution with mean and SD determined by the four points in each bin. Similarly, we also averaged the Hand-Target difference, the explicit eye, and the implicit eye in the four trials preceding each mini exclusion block. We used these to calculate the "explicit exclusion" by subtracting the binned implicit exclusion from the binned Hand-Target difference before the mini exclusion block (Table 1). We defined the "implicit difference" as the difference between the binned implicit eye before the mini exclusion block and the implicit exclusion. Since, for some subjects, some bins were missing, we used probabilistic principal components analysis (PPCA) (Tipping and Bishop, 1999) to fill in the missing values by projecting the data (6 bins/subject) onto the first five principal 


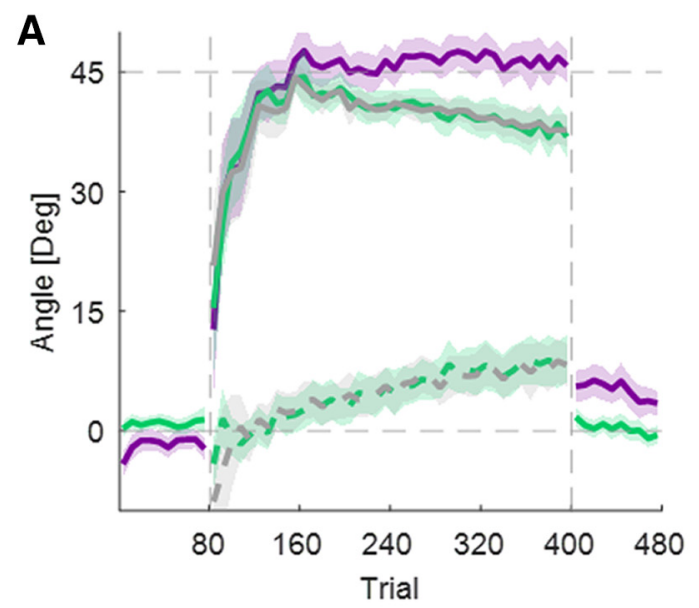

C

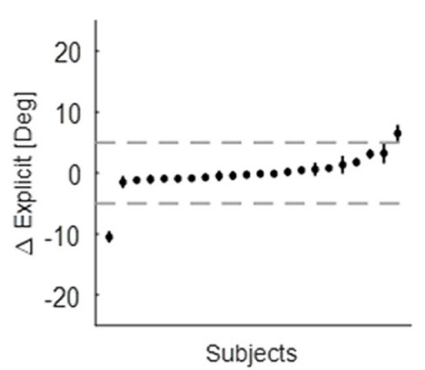

D

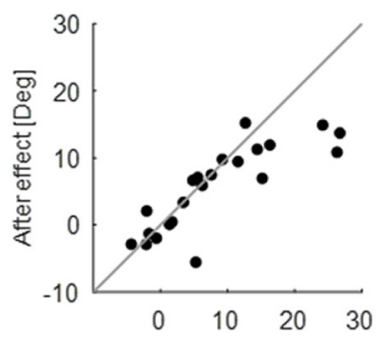

Implicit Report End of Rotation [Deg]
B

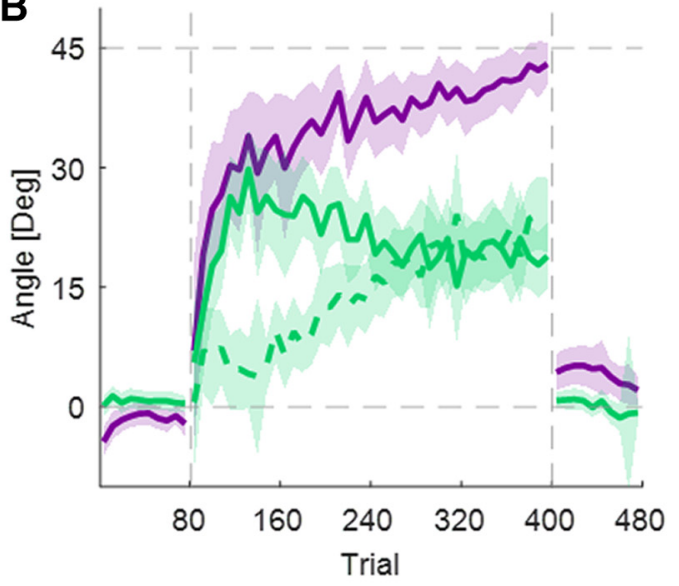

Hand Explicit Report Implicit Report Explicit Eye Implicit Eye

Figure 3. Experiment 1. A, Learning curves of the report group. $\boldsymbol{B}$, Learning curves of the no-report group. $\boldsymbol{C}$, Subjects' differences between the explicit report and the explicit eye. Each dot is the averaged difference between the explicit report and the explicit eye during the rotation block, and each line shows the SEM of this difference. Dashed gray lines show a $5^{\circ}$ region of equivalence. $\boldsymbol{D}$, The relation between the aftereffect and the implicit report at the end of adaptation in the report group. $\boldsymbol{E}$, The relation between the aftereffect and the implicit eye at the end of adaptation in the report group. $\boldsymbol{F}$, The relation between the aftereffect and the implicit eye at the end of adaptation in the no-report group. $\boldsymbol{D}-\boldsymbol{F}$, Dots denote individual subjects; identity line is in black.

components (PCs) space and then back to the original 6-dimensional space.

In the reaction time analysis, we binned the HandTarget difference and the explicit eye according to the RT. We divided the reaction time into windows of $25 \mathrm{~ms}$ from 0 to $2000 \mathrm{~ms}$ and averaged over the Hand-Target difference and the explicit eye of all trials with RT in the same window. The SEM for each window was calculated by dividing the SD of each bin by the square root of the number of trials in this bin.

In all learning curves, we focused on three phases: the "initial rise" was characterized by the third bin in the rotation block (trial numbers 97-104; rotation trials 17$24) ;$ the "late early rise" was characterized by the 10th bin in the rotation block (trial numbers 153-160; rotation trials 73-80), and the "end of adaptation" was characterized by the last bin of the rotation block (trial numbers 393-400; rotation trials $313-320$ ).

\section{Clustering}

In the second experiment, we clustered the subjects with fuzzy c-means (FCM) clustering. We tested clustering for two through six clusters. Following Haar et al. (2015a), we used the cluster validity index proposed by Zhang et al. (2008). This index uses a ratio between a variation measure in each cluster and a separation measure be- tween the fuzzy clusters. The smaller the ratio, the better the clustering. Clustering was applied in two steps: the first on the six binned values of implicit difference; the second on the explicit eye during rotation trials. For each cluster found in the first step, perform additional clustering on the first three PCs from the 40 bins of rotation trials.

\section{Results}

We recorded eye movements that subjects made during hand-reaching movements perturbed by a clockwise visuomotor rotation to study the relation between subjects' gaze and explicit learning. In the first experiment, we developed and validated our explicit eye measure, and, in the second experiment, we explored the time course of the explicit eye measure and its relation to exclusion, a measure of implicit learning.

\section{Experiment 1}

The first experiment followed the protocol of Taylor et al. (2014) and compared a group adapting while reporting (R) with one that did not report (NR). Both groups showed an initial rapid rise in the Hand-Target difference (Fig. $3 A, B$ ). The initial rise was $32.9 \pm 6.2^{\circ}$ and $24.7 \pm 8.5^{\circ}$ for the $R$ and NR groups, respectively. The difference between the groups in the initial rise was $8.2 \pm 10.5^{\circ}$. After the initial rise, adaptation continues albeit more slowly. This 
slower rise continues until subjects have zero error. The report group plateaued after $\sim 80$ trials with mean HandTarget difference of $46.5 \pm 2.8^{\circ}$ at the late early rise. The NR group was slower to reach plateau and at the late early rise their mean Hand-Target difference was only $33.9 \pm$ $5.4^{\circ}$. The difference between groups in this late early adaptation phase is $12.6 \pm 6.1^{\circ}$. By the end of adaptation phase, the mean Hand-Target difference is $45.8 \pm 2.1^{\circ}$ and $43.0 \pm 2.7^{\circ}$ in the $R$ and NR groups, respectively.

Explicit report and explicit eye in both groups rise quickly and fall off slowly, reflecting a slow but steady increase in implicit knowledge. Differences between groups in the explicit eye at the initial rise (difference, $15.8 \pm 8.6^{\circ}$ ) and in the late early rise (difference, $19.5 \pm 6.0^{\circ}$ ) mirrors the difference in the Hand-Target difference, reflecting the dominant role of explicit knowledge in initial learning across both groups. By the end of adaptation, the difference between the $\mathrm{R}$ and NR groups still exists $\left(18.0 \pm 4.8^{\circ}\right)$.

In the R group, explicit eye and explicit report match with an average difference of $-0.02 \pm 0.8^{\circ}$. This is true not only on average, but also for each subject (Fig. 3C). As a consequence, the corresponding implicit measures also match. The average difference is $-0.3 \pm 0.8^{\circ}$, and this is consistent with the aftereffect measure of the implicit: the difference between the aftereffects and the implicit components is $3.4 \pm 3.0^{\circ}$ (Fig. $3 D, E$ ). This contrasts with the consistent lack of a match between implicit eye and aftereffect in the NR group (Fig. 3F). For the NR group, the implicit eye measure suggested a much larger implicit component than revealed by the aftereffect, where the difference between the aftereffects and the implicit eye was $19.6 \pm 4.5^{\circ}$. Nevertheless, the aftereffect and the implicit eye correlated in both groups, with mean Spearman correlations of $0.75 \pm 0.09$ and $0.67 \pm$ 0.11 for the R and NR groups, respectively. This suggests the possibility of a shared component, despite the difference in average values.

\section{Experiment 2}

To explore the difference between eye movements and the aftereffect, we conducted a second experiment where we added exclusion trials during the rotation block. Those trials were no visual feedback trials in which we asked subjects to ignore their strategy and aim directly at the target. Since, by aiming at the target, they remove the expression of their explicit knowledge, the remaining HandTarget difference reflects only their implicit learning. Five mini-blocks of four exclusion trials were used during the rotation, and these were combined with a virtual miniblock of the first four exclusion trials of the aftereffect. This led to a total of 24 exclusion trials in six mini-blocks for four trials each.

As in the NR group of the first experiment, the HandTarget difference initially rose quickly, and later continued to rise slowly (Fig. 4A). In this experiment, on average, subjects did not reach full adaptation. Here, too, the explicit eye initially rises quickly. However, it did not decline as much as in the first experiment. The implicit eye also rose slowly, but less than in the first experiment. (This is a computational consequence of the reduction in the explicit eye mentioned above. The implicit eye is simply
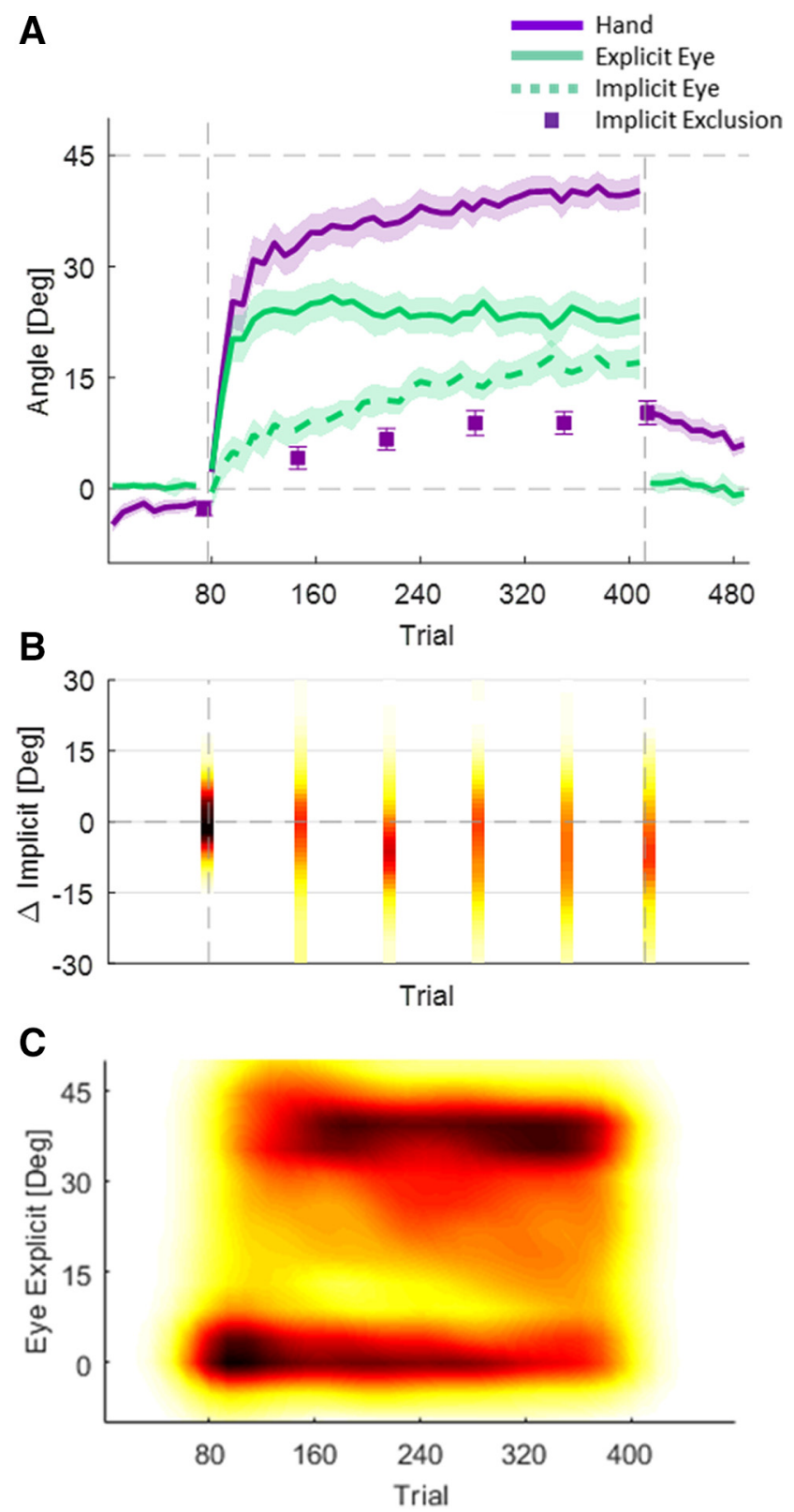

Figure 4. Experiment 2. A, Averaged learning curves across the entire group. Error bars in the implicit exclusion and the shaded area represent the SEM. B. Distribution of the differences between the two implicit measures. $\boldsymbol{C}$, Distribution of the explicit eye.

the difference between Hand-Target difference and explicit eye.) Replicating experiment 1, there was a gap between the implicit exclusion and the implicit eye. On average, the Hand-Target difference showed an initial rise of $25.2 \pm 3.7^{\circ}$. By the end of adaptation, the Hand-Target difference reached $40.2 \pm 2.2^{\circ}$. The explicit eye, on average, had an initial rise of $20.2 \pm 3.1^{\circ}$ and stabilized at this level until the end of the adaptation, where it reached $23.3 \pm 2.7^{\circ}$. The implicit exclusion was lower than the implicit eye, with an average gap of $5.4 \pm 1.3^{\circ}$. However, this gap was not consistent across subjects (Fig. 4B). For some subjects, almost no gap existed, while, for others, it was quite substantial. 

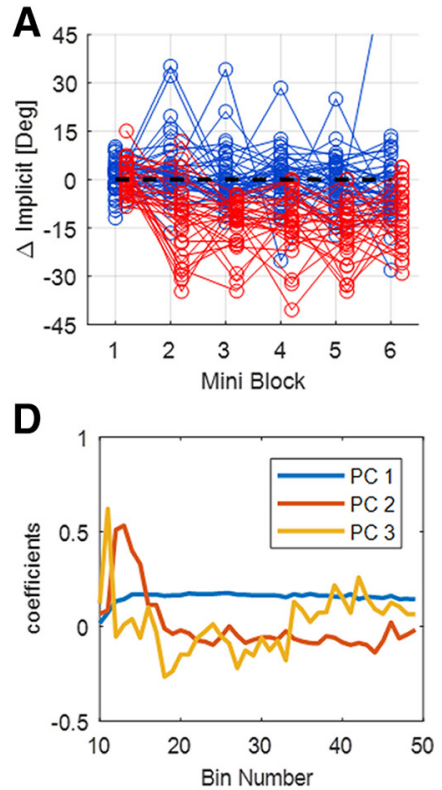

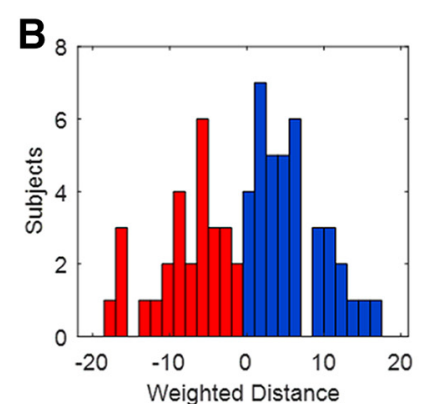

E

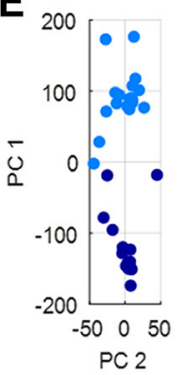

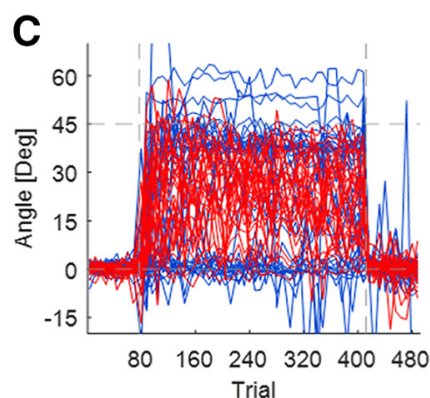

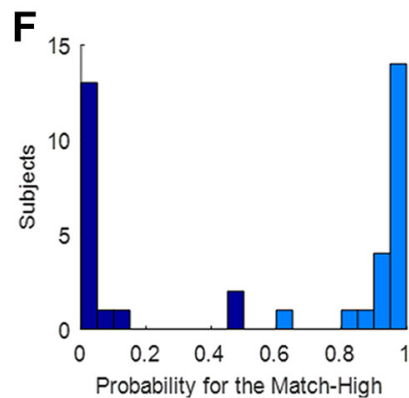

Figure 5. Experiment 2: clustering steps. $\boldsymbol{A}-\boldsymbol{C}$, Clustering step 1. Blue lines are subjects who belong to the cluster in which the implicit measures match, and red lines are those who belong to the cluster in which the implicit measures do not match. $\boldsymbol{A}$, The difference between the two implicit measures for the six mini-blocks of exclusion. Each line depicts an individual subject. $\boldsymbol{B}$, Histogram of the weighted averaged implicit difference. $\boldsymbol{C}$, Explicit eye for all subjects. $\boldsymbol{D}-\boldsymbol{F}$, Clustering step 2. $\boldsymbol{D}$, Three principal components on which the data are clustered are shown. $\boldsymbol{E}$, The first and the second PCs. Dots represent individual subjects: light blue dots are subjects with high explicit eye, and dark blue dots are subjects with low explicit eye. $\boldsymbol{F}$, Histogram of the probability of subjects to belong to the high explicit eye cluster.

Additionally, subjects who did not show a gap between implicit eye and implicit exclusion seemed to be of two types. For some, gaze stayed on the target, reflecting an explicit eye adaptation of $0^{\circ}$ (these subjects also had a very slow rise in Hand-Target difference, which reached no more than $30^{\circ}$ ). For others, gaze shifted to the HandTarget early in adaptation, reflecting nearly full explicit knowledge (Fig. 4C). Subjects in this latter group also had a very quick initial rise in the Hand-Target difference. These results suggest extensive explicit adaptation in these subjects.

Having noticed this pattern in the data, we clustered the subjects accordingly (Fig. 5). We used a two-step approach. In the first step, we clustered subjects using FCM according to the difference between the two measures of implicit. The cluster validity index (Zhang et al., 2008) values suggested two clusters. As expected, for one cluster implicit eye and implicit exclusion matched, while for the other cluster implicit exclusion was greater than implicit eye. We called these the "matched-implicit" and "No-Match" clusters. In the second clustering step, we further clustered the matched-implicit cluster according to the explicit eye measured throughout the entire rotation block. We used PPCA to reduce the dimensionality of the data. We ran FCM clustering over the first three components, which captured $93 \%$ of the overall variance. The cluster validity values again suggested two clusters. Applying the same clustering approach to the No-Match group did not reveal any evidence of clustering.

The two-step clustering method found three clusters of subjects. Two of them are derived from the matchedimplicit cluster of the first step, and, for them, the explicit eye faithfully reflects explicit knowledge. From those two, we called the one that had more explicit adaptation "Match-High" $(n=21)$. The Hand-Target difference of these subjects rose quickly to $31.8 \pm 6.6^{\circ}$ during the initial rise, and then to $45.4 \pm 2.2^{\circ}$ at the late early rise, and to $48.7 \pm 2.4^{\circ}$ at the end of adaptation (Fig. $6 A$ ). This group of subjects counteracts the rotation fully and quickly using an explicit strategy. This can be seen in the explicit eye, which rose quickly to $31.0 \pm 6.2^{\circ}$ and later to $41.6 \pm 2.7^{\circ}$, and by the end of adaptation was slightly reduced to $38.8 \pm 2.3^{\circ}$ (Fig. 6B). Their implicit learning, measured by implicit eye, was $10.6 \pm 2.8^{\circ}$ at the end of the rotation block (Fig. 6C). For this group, explicit eye and implicit eye matched explicit exclusion and implicit exclusion (differences of $0.5 \pm 1.1^{\circ}$ and $-0.3 \pm 1.6^{\circ}$, respectively).

In contrast, the other matching cluster had very little explicit adaptation. We called it "Match-Low" $(n=17)$. For these subjects, the difference between explicit eye and explicit exclusion was also very small $\left(-0.1 \pm 1.9^{\circ}\right)$, as was the case for implicit eye and implicit exclusion $\left(-0.5 \pm 2.4^{\circ}\right)$. The lack of explicit strategy is reflected in the very low explicit eye, which was $3.0 \pm 4.2^{\circ}$ in the initial rise, $5.8 \pm 4.0^{\circ}$ at the late early rise, and reached $7.1 \pm$ $5.9^{\circ}$ at the end of adaptation (Fig. 6B). Since this cluster used no explicit strategy, the subjects adapt to the rotation only implicitly, and thus have very slow adaptation (Fig. 6A). The average Hand-Target difference changed from $3.6 \pm 5.6^{\circ}$ at the initial rise to $12.7 \pm 4.7^{\circ}$ at the late early rise and reached $22.7 \pm 4.3^{\circ}$ at the end of the rotation block. Hence, the Match-High and Match-Low groups had very different Hand-Target difference and explicit eye. The implicit eye of the Match-Low group 

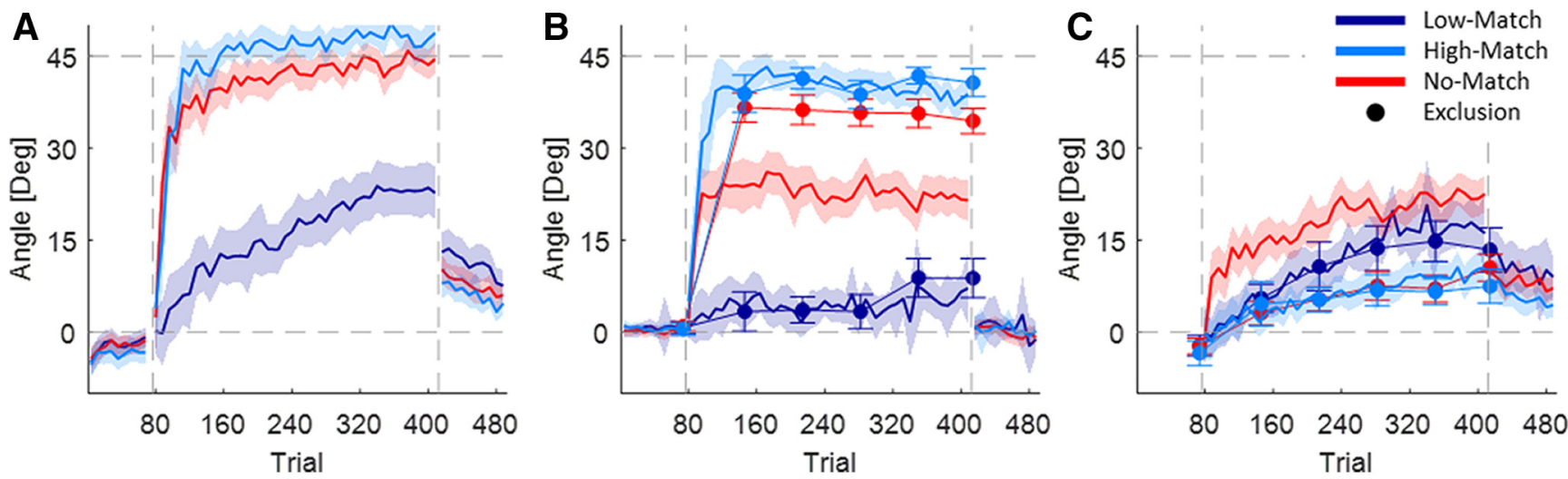

Figure 6. Experiment 2: learning curves per cluster. $\boldsymbol{A}$, Hand-Target difference for the Match-Low, Match-High and No-Match clusters. B, Explicit eye (lines) and explicit exclusion (circles) for the three clusters. $\boldsymbol{C}$, Implicit eye (lines) and implicit exclusion (circles) for the three clusters. Error bars around the implicit exclusion and the shaded area represent the SEM.

reached $16.1 \pm 6.5^{\circ}$ by the end of the rotation block, $5.5 \pm 7.1^{\circ}$ higher than that of the Match-High group.

The No-Match cluster $(n=28$; which was the second cluster in step 1) was characterized by a lack of correspondence between implicit eye and implicit exclusion (Fig. 6C). Accordingly, this cluster adapts to the rotation using a mostly explicit strategy (Fig. 6B). However, the explicit eye captures only part of the explicit learning measured with explicit exclusion. The difference between them, across subjects, is $13.5 \pm 1.5^{\circ}$ by the end of the rotation block. The fact that explicit adaptation does exist in these subjects is supported by the rapid rise of the Hand-Target difference: $33.5 \pm 5.0^{\circ}$ in the initial rise, $39.8 \pm 2.8^{\circ}$ at the late early rise, and $44.5 \pm 2.5^{\circ}$ at the end of adaptation. This performance is similar to that of the Match-High group, with differences of $-1.7 \pm 8.2^{\circ}$, $5.6 \pm 3.6^{\circ}$, and $4.2 \pm 3.5^{\circ}$ at the initial rise, at the late early rise, and at the end of adaptation. In contrast, the explicit eye of this No-Match group was very different from that of the Match-High. It changed from $22.6 \pm 4.1^{\circ}$ in the initial rise to $24.1 \pm 3.6^{\circ}$ at the late early rise and to $21.5 \pm 3.3^{\circ}$ by the end of adaptation. Both measures of implicit of the No-Match cluster rose slowly; the implicit eye was much higher than implicit exclusion and the difference was $13.3 \pm 1.7^{\circ}$ by the end of the rotation block.

Following previous studies that suggested that explicit learning requires longer RTs (Benson et al., 2011; Leow et al., 2017; McDougle and Taylor, 2019), we looked for differences in the RT between our different explicit learning groups (Fig. 7A). The Match-High group showed the longest RTs. Their RTs decreased by $758 \pm 370 \mathrm{~ms}$ from initial adaptation to the end of adaptation, which is in line with the decrease in their explicit eye over the adaptation period. The RTs of those in the Match-Low group, who had almost no explicit adaptation, were faster. Though the explicit eye of the Match-Low group increased during adaptation, their RTs decreased by $450 \pm 341 \mathrm{~ms}$. The RTs of the No-Match group started as fast as those of the Match-Low group, and they grew quicker by $272 \pm 228$ ms. Interestingly, the Match-High group had longer RTs in the baseline and the washout blocks as well. The RTs of this group were different from those of the low-match and the No-Match groups by $249 \pm 181$ and $404 \pm 152 \mathrm{~ms}$, respectively. It appears that behavior in the clusters is different even before adaptation begins.

Figure 7, $B$ and $C$, shows the relationship between RT and Hand-Target difference and between RT and explicit eye. For RTs $>400 \mathrm{~ms}$, larger RTs were associated with larger Hand-Target differences and with more explicit eye. This relationship saturated in the Match-Low group for RTs longer than $700 \mathrm{~ms}$. While the results are partially consistent with the hypothesis that explicit eye reflects aspects of explicit knowledge, we also expected that averaged RTs in the No-Match group would be closer to
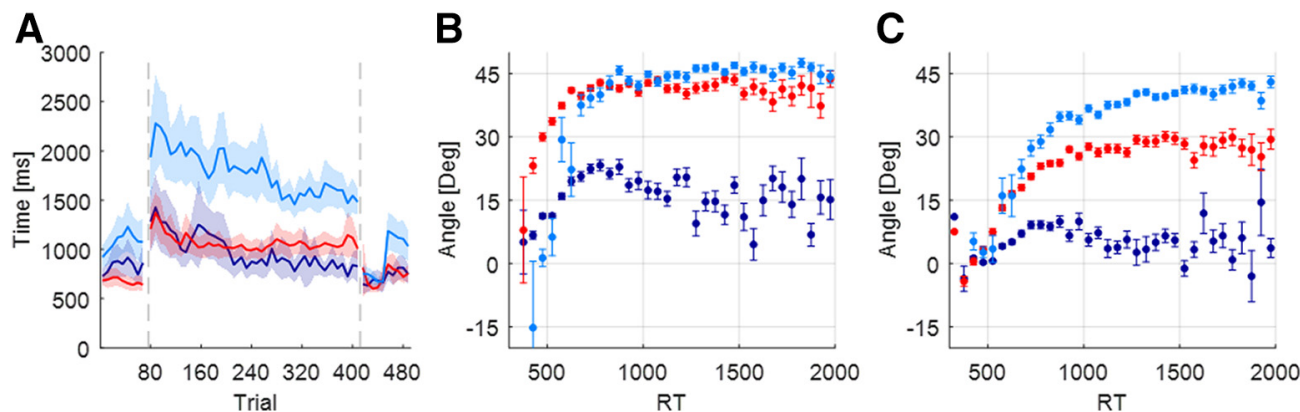

Figure 7. Experiment 2: reaction times. $\boldsymbol{A}$, Averaged reaction time for each cluster. The shaded area represents the SEM. $\boldsymbol{B}, \boldsymbol{C}, \mathrm{Cluster}$ means of Hand-Target differences $(\boldsymbol{B})$ and cluster means of explicit eye as a function of RT (binned in 25 ms bins; $\boldsymbol{C}$ ). Each dot is a bin, and error bars represent the SEM of each bin. 
those of the Match-High group than to those of the Match-Low group. In fact, Figure $7 A$ shows that the opposite is the case. For this group, the explicit eye reflects only one component of explicit learning, the same one that is correlated to the RTs, while there is another explicit component that is not related to either eye movements or RTs.

\section{Discussion}

In this study, we explored the extent to which explicit components of visuomotor adaptation are reflected in eye movements. We did this by comparing eye movements to two accepted measures of explicit learning: verbal report and the exclusion test. Our experiments showed that eye movements have a stable pattern: after target appearance, the eyes saccade from the origin to the target, and then, before movement onset, the eyes saccade again in the direction toward which the subject will aim. We believe that these eye movements provide a measure of explicit adaptation (we called it explicit eye); however, this measure only reflects part of the explicit adaptation. Our first experiment showed that when subjects report their intended direction, explicit eye and the other two measures (verbal report and exclusion) all matched. In contrast, when subjects did not report, explicit eye only reflected part of the explicit adaptation, as reflected in the exclusion. However, the two were correlated, and this suggests that explicit eye might be reflecting select components of the explicit exclusion. In our second experiment, we tried to explore more fully the time course of the separation of explicit eye from explicit shown by exclusion. We found that the two diverge early in adaptation. In analyzing the data of the second experiment, we found three groups of subjects. The first group adapted fully to the rotation and had eye movements consistent with performance in exclusion trials (Match-High group); the second group also adapted fully but had less explicit eye than would be expected from exclusion trials (No-Match group); the third group only adapted partially and had eye movements consistent with lack of explicit adaptation in the exclusion trials (Match-Low group). The learning curves of this last group were similar to those reported in paradigms where subjects had only implicit adaptation (Morehead et al., 2017; Kim et al., 2018, 2019).

Smith et al. (2006) proposed a two-state model of motor adaptation that is still the most widely used model in the field. It has been nicely mapped onto explicit (fast) and implicit (slow) components of adaptation (McDougle et al., 2015). However, there have been suggestions that there are more than two states in the adaptation process, and that there may be multiple explicit and implicit components, potentially with different time constants. Forano and Franklin (2019) showed that dual adaptation can be best explained by models with two fast components, and McDougle and Taylor (2019) showed two explicit strategies in visuomotor rotation: caching and mental rotation. Presumably, in our study, the group for which explicit eye explained only part of the explicit learning (No-Match group) used multiple explicit strategies, while the group where measures of explicit learning matched used one
(Match-High group) or, perhaps, none (Match-Low group).

The question arises whether the components of the explicit adaptation that are reflected in explicit eye map to the explicit strategies identified by McDougle and Taylor (2019). In that study, the key difference in the strategies was that one strategy introduced a correlation between rotation and reaction time while the other did not. Consequently, we examined reaction times in the different groups. We found that the group with the single explicit strategy (captured by gaze; Match-High group) had very long reaction times relative to the other groups. Interestingly, these subjects had longer reaction times in the baseline phase as well, suggesting that they were more carefully and explicitly controlled movers even during normal movement. The reaction times of the No-Match group were much lower. That is, the No-Match group achieved explicit adaptation comparable to that of the Match-High group, but their explicit adaptation required less preparation time. The Match-Low subjects, who only adapted implicitly, had the fastest reaction times. Together, we hypothesize that the explicit components reflected in explicit eye are the same components that drive longer reaction times. McDougle and Taylor (2019) identified this as the process of mental rotation and contrasted it with the low-reaction time mechanism of caching.

Links between the intended direction of movement and eye movements have been foreshadowed (Rentsch and Rand, 2014; Rand and Rentsch, 2016) and demonstrated explicitly (de Brouwer et al., 2018). Our study supports these earlier findings, although there are some technical issues that deserve consideration. First, we followed the Rand and Rentsch (2016) study in using only end-point feedback rather than continuous presentation of the cursor. This simplified the eye movements and allowed us to determine that the fixations immediately before movement initiation provided the most reliable estimate of explicit adaptation. The specific timing at which eye movements are considered has consequences. Our findings match those of de Brouwer et al. (2018) in that both studies find that eye movements reflect explicit adaptation. An important difference in the findings relates to the timing at which eye movements are considered. de Brouwer et al. (2018) evaluate the fixation closest to the rotation angle. Our data support the basic statistical logic that such a measure will tend to be biased. The last fixation before movement onset was a more stable measure and is consistent with earlier results on the specific timing with which eye movements predict hand movements (Ariff et al., 2002). This measure also allowed an unbiased quantification of explicit adaptation even in subjects with very little explicit adaptation, which is key for identifying the Match-Low group. This difference in the measures may be one reason why de Brouwer et al. (2018) did not identify the three different groups of subjects we found.

Last, we note that each of our measures (report, eye, and exclusion) measured either explicit adaptation or implicit adaptation, but not both. We then calculated the complementary adaptation by subtracting the measured component from the hand direction. Much influential re- 
search in the field takes this approach: it assumes that hand direction is the simple sum of an explicit and an implicit component (Taylor et al., 2014; Huberdeau et al., 2015; McDougle et al., 2015; Christou et al., 2016; Leow et al., 2017). However, this assumption has been questioned, and various efforts to validate it have been put forward including the use of inclusion trials in combination with exclusion trials (Werner et al., 2015; Neville and Cressman, 2018; Modchalingam et al., 2019). Exclusion trials test for explicit knowledge by asking subjects to stop using what they know. Inclusion trials verify this ability to explicitly control behavior by asking subjects to go back to using what they know. Since, in most studies, inclusion trials show less adaptation than do the rotation trials that preceded them, it seems that the total behavior must involve some component that is more easily turned off than turned back on. This is in line with the claim in this article that explicit knowledge may involve multiple components. We use inclusion trials to further explore this idea in the study by Maresch and Donchin (2019).

This study provides replicates and extends earlier findings that eye movements reflect an explicit strategy in visuomotor adaptation. It supports other reports demonstrating multiple explicit components in adaptation. It seems that some components of explicit adaptation are not reflected in the eye movements. The components reflected in the eye movements are correlated with reaction time and may include the component identified by McDougle and Taylor (2019) as mental rotation. While eye movements may not be a perfect measure of explicit adaptation, they could be used to capture this component on a trial-by-trial basis without influencing the adaptation.

\section{References}

Amrhein V, Greenland S, McShane B (2019) Scientists rise up against statistical significance. Nature 567:305-307.

Ariff G, Donchin O, Nanayakkara T, Shadmehr R (2002) A real-time state predictor in motor control: study of saccadic eye movements during unseen reaching movements. J Neurosci 22:7721-7729.

Benson BL, Anguera JA, Seidler RD (2011) A spatial explicit strategy reduces error but interferes with sensorimotor adaptation. J Neurophysiol 105:2843-2851.

Christou Al, Miall RC, McNab F, Galea JM (2016) Individual differences in explicit and implicit visuomotor learning and working memory capacity. Sci Rep 6:36633.

de Brouwer AJ, Albaghdadi M, Flanagan JR, Gallivan JP (2018) Using gaze behavior to parcellate the explicit and implicit contributions to visuomotor learning. J Neurophysiol 120:1602-1615.

Forano M, Franklin DW (2019) Timescales of motor memory formation in dual-adaptation. bioRxiv. Advance online publication. Retrieved November 26, 2019. doi:10.1101/698167.

Galea JM, Mallia E, Rothwell J, Diedrichsen J (2015) The dissociable effects of punishment and reward on motor learning. Nat Neurosci 18:597-602.

Ghilardi M, Ghez C, Dhawan V, Moeller J, Mentis M, Nakamura T, Antonini A, Eidelberg D (2000) Patterns of regional brain activation associated with different forms of motor learning. Brain Res 871: 127-145.

Haar S, Givon-Mayo R, Barmack NH, Yakhnitsa V, Donchin O (2015a) Spontaneous activity does not predict morphological type in cerebellar interneurons. J Neurosci 35:1432-1442.

Haar S, Donchin O, Dinstein I (2015b) Dissociating visual and motor directional selectivity using visuomotor adaptation. J Neurosci 35:6813-6821.
Haith AM, Krakauer JW (2018) The multiple effects of practice: skill, habit and reduced cognitive load. Curr Opin Behav Sci 20:196201.

Hegele M, Heuer H (2010) Implicit and explicit components of dual adaptation to visuomotor rotations. Conscious Cogn 19:906-917.

Huberdeau DM, Krakauer JW, Haith AM (2015) Dual-process decomposition in human sensorimotor adaptation. Curr Opin Neurobiol 33:71-77.

Kim HE, Morehead JR, Parvin DE, Moazzezi R, Ivry RB (2018) Invariant errors reveal limitations in motor correction rather than constraints on error sensitivity. Commun Biol 1:19.

Kim HE, Parvin DE, Ivry RB (2019) The influence of task outcome on implicit motor learning. Elife 8:e39882.

Krakauer JW, Ghilardi M, Ghez C (1999) Independent learning of internal models for kinematic and dynamic control of reaching. Nat Neurosci 2:1026-1031.

Leow L-A, Gunn R, Marinovic W, Carroll TJ (2017) Estimating the implicit component of visuomotor rotation learning by constraining movement preparation time. J Neurophysiol 118:666-676.

Maresch J, Donchin O (2019) Reporting affects explicit knowledge in visuomotor rotations in ways not measured by reporting. bioRxiv. Advance online publication. Retrieved November 29, 2019. doi: $10.1101 / 702290$

Mazzoni P, Krakauer J (2006) An implicit plan overrides an explicit strategy during visuomotor adaptation. J Neurosci 26:3642-3645.

McDougle SD, Taylor JA (2019) Dissociable cognitive strategies for sensorimotor learning. Nat Commun 10:40.

McDougle SD, Bond KM, Taylor JA (2015) Explicit and implicit processes constitute the fast and slow processes of sensorimotor learning. J Neurosci 35:9568-9579.

Modchalingam S, Vachon CM, 't Hart BM, Henriques DYP (2019) The effects of awareness of the perturbation during motor adaptation on hand localization. PLoS One 14:e0220884.

Morehead JR, Taylor JA, Parvin DE, Ivry RB (2017) Characteristics of implicit sensorimotor adaptation revealed by task-irrelevant clamped feedback. J Cogn Neurosci 29:1061-1074.

Neville K-M, Cressman EK (2018) The influence of awareness on explicit and implicit contributions to visuomotor adaptation over time. Exp Brain Res 236:2047-2059.

Rabe K, Livne O, Gizewski ER, Aurich V, Beck A, Timmann D, Donchin O (2009) Adaptation to visuomotor rotation and force field perturbation is correlated to different brain areas in patients with cerebellar degeneration. J Neurophysiol 101:1961-1971.

Rand MK, Rentsch S (2016) Eye-hand coordination during visuomotor adaptation with different rotation angles: effects of terminal visual feedback. PLoS One 11:e0164602.

Rentsch S, Rand MK (2014) Eye-hand coordination during visuomotor adaptation with different rotation angles. PLoS One 9:e109819.

Smith MA, Ghazizadeh A, Shadmehr R (2006) Interacting adaptive processes with different timescales underlie short-term motor learning. PLoS Biol 4:e179.

Taylor JA, Ivry RB (2011) Flexible cognitive strategies during motor learning. PLoS Comput Biol 7:e1001096.

Taylor JA, Ivry RB (2013) Context-dependent generalization. Front Hum Neurosci 7:1-14.

Taylor JA, Krakauer JW, Ivry RB (2014) Explicit and implicit contributions to learning in a sensorimotor adaptation task. J Neurosci 34:3023-3032.

Tipping ME, Bishop CM (1999) Probabilistic principal component analysis. J R Stat Soc Series B Stat Methodol 61:611-622.

Werner S, Van Aken BC, Hulst T, Frens MA, Van Der Geest JN, Strüder HK, Donchin O (2015) Awareness of sensorimotor adaptation to visual rotations of different size. PLoS One 10:1-18.

Wong AL, Marvel CL, Taylor JA, Krakauer JW (2019) Can patients with cerebellar disease switch learning mechanisms to reduce their adaptation deficits? Brain 142:662-673.

Zhang Y, Wang W, Zhang X, Li Y (2008) A cluster validity index for fuzzy clustering. Inf Sci 178:1205-1218. 